\title{
Spontaneous Tribocharging of Similar Materials
}

\author{
Troy Shinbrot* \& Qiao Zhao \\ Dept. of Biomedical Engineering \\ Rutgers University, Piscataway, NJ 08816 \\ shinbrot@soemail.rutgers.edu :: (732) 445-4500 x6310
}

\begin{abstract}
:
In this letter, we investigate the spontaneous triboelectrification of similar materials. This effect, first reported in 1986, has been little studied but is easily reproduced. We report here using a model system of common balloons that identical insulators, prepared in the same way and rubbed symmetrically, break symmetry so that one balloon becomes positive and the other negative. Curiously, the distribution of charges on the balloons appear to be self-similar, with different charge patterns on the positive and the negative surface. We propose a mechanism in which an initial localized charge may spawn the production of smaller localized charges of the same polarity, and we speculate that a similar charging instability may be at work in other charging phenomena.
\end{abstract}

PACS Numbers: 01.50.Wg, 45.70.Qj, 05.45.Df, 46.55.+d, 52.80.-s 


\section{Introduction:}

It has been recognized at least since the $16^{\text {th }}$ century ${ }^{1,2}$ that insulators (originally termed "electrics") can readily be charged by rubbing or contact, while conductors ("non-electrics") are more difficult to charge. Careful experiments have shown this effect to be distinct from the ability of conductors to carry acquired charges to ground ${ }^{3,4}$. Thus PTFE - one of the best insulators known - is the material of choice to produce megaVolt potentials in Van de Graaff generators. This is paradoxical since insulators lack free charge carriers, and it has remained unexplained to this day how they recruit these charges. The paradox has been compounded by experiments performed in the 1980's that demonstrated that even identical insulators can transfer charges during rubbing ${ }^{5}$. In one sense, this should come as little surprise, since charge is also generated through contact between identical materials in the most widely experienced electrification system: atmospheric lightning. Similarly, large charges are acquired by Aeolian transport of desert sands, which have little to rub against beyond other sand ${ }^{6}$. In a more fundamental sense, however, it is troublesomely unclear how insulators in general acquire the free charges that they convey during tribocharging, and how identical insulators in particular can charge one another at all.

In this letter, we investigate the mechanism by which charges can be acquired and amplified by rubbing of identical insulators. Unexpectedly, we find that self-similar charge distributions appear spontaneously on the rubbed surfaces, and we find that there is evidence to suggest that this occurs through a fundamental instability by which localized charged regions beget further charges of the same sign. We find, furthermore, that the proposed instability appears to recruit charge carriers from the environment - i.e. from the surrounding atmosphere - rather than by transforming the intrinsic insulating nature of the material. 


\section{Results and discussion:}

To examine the tribocharging of similar insulators, we choose a model system that is easily reproduced: we rub common latex balloons symmetrically against one another, as shown in Fig. 1(a). The balloons are obtained from the same lot, are the same color, are inflated to the same size, are initially washed with alcohol to remove surface oils or dirt, and are rubbed together symmetrically in a circular motion. As demonstrated in Fig. 1(a) (and online in Supplemental Materials; for review placed at: http://coewww.rutgers.edu/ shinbrot/temp/TriboBalloons.html), the balloons spontaneously charge one another sufficiently to suspend one balloon from the other against its weight. Measurements of electric potentials nearby the balloons using the voltmeter probe reveal that the balloons are initially charge-neutral, but following rubbing break symmetry to generate a net charge, with one becoming highly positive $(>3 \mathrm{kV}$ at $5 \mathrm{~cm}$ distance) the other equally negative. The choice of balloon polarity appears to be random: we clean the balloons with alcohol (which neutralizes the charges), and once dried we rub the same balloons together and obtain new charges that show no apparent correlation with the prior polarity.
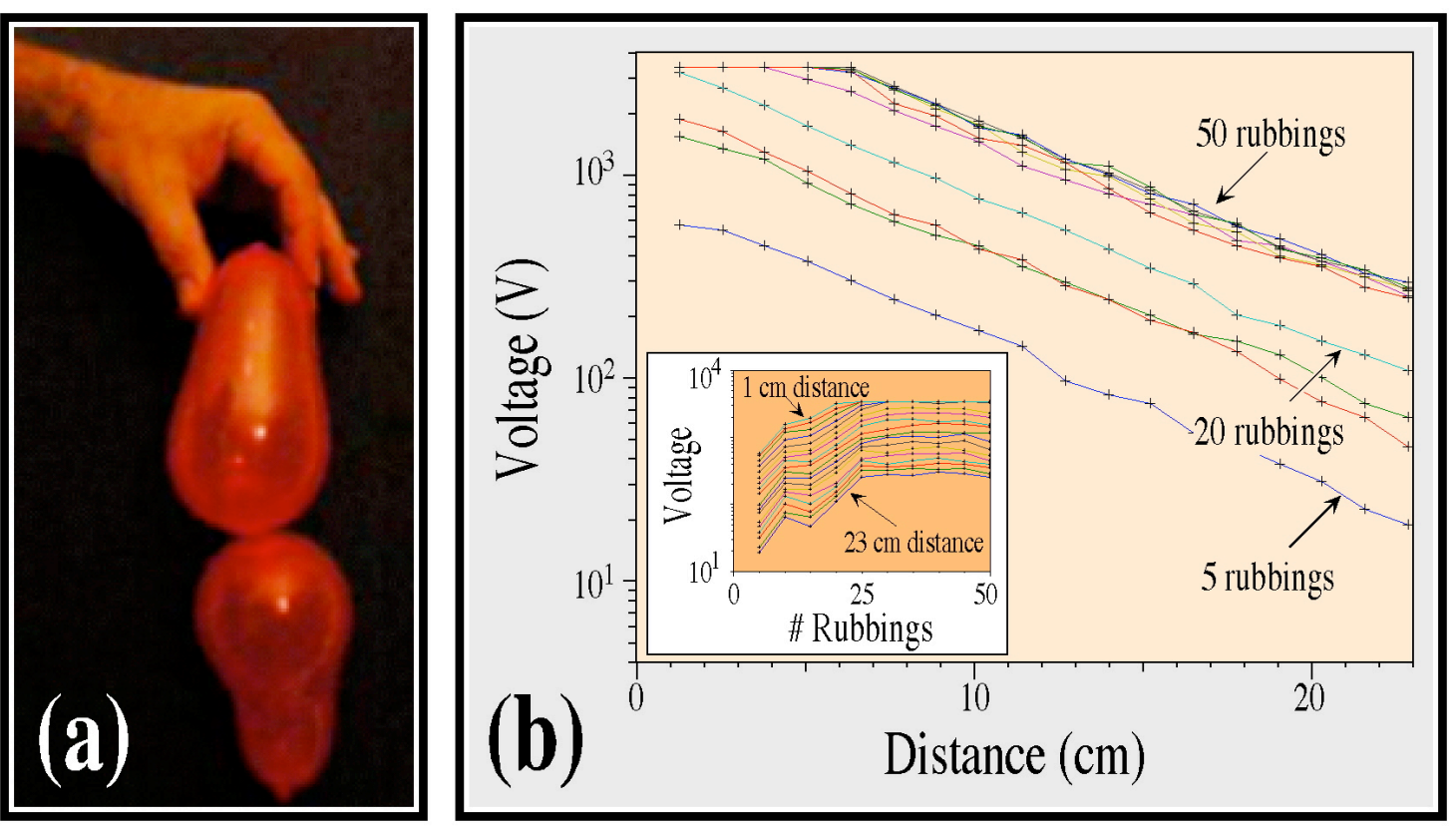

Figure 1 -(a) Identical pairs of latex balloons rubbed against one another adhere electrostatically; movies of this effect appear at http://coewww.rutgers.edu/ shinbrot/temp/TriboBalloons.html. The balloons invariably break symmetry so that one becomes positive and the other negative. (b) Measurements of voltage as a function of distance from a positively charged balloon vs. distance from balloon to probe and (inset) number of times the balloons are rubbed against one another. Data from negatively charged balloon (not shown) are similar except for sign. One "rubbing" in these measurements consists of rotating the balloons back and forth once while pressing the balloons together firmly; distances are measured from the closest point on the balloon to the probe tip. Inset shows growth in voltage as a function of number of rubbings at numerous fixed positions.

We emphasize that in order for identical balloons to tribocharge one another, positive charges must move from a more electro-negative surface to a more electro-positive one against the apparent 
electrostatic gradient imposed by Coulomb's law. We quantify the acquired charge distributions in two ways.

First, we directly measure the voltage on each balloon as a function of distance from each balloon and the number of times the balloons have been rubbed together, using a null voltmeter probe (details in Materials and Methods). The voltage is expected to decrease as $a_{n} / r^{n}$, for a multipole of moment $n$, where $a_{n}$ is the magnitude of the n-pole's contribution and $r$ is the distance to the center of the charge distribution ${ }^{7}$. To make these measurements, we attach the probe to a fixed stand distant from other charges or conductors and slowly move the balloon toward the probe, recording the position and probe voltage with a video camera. Against expectation, the voltage does not decrease as a power law, but as shown in Fig. 1(b), decreases exponentially: fits of the data shown to an exponential function have correlation coefficients between 0.998 and 0.999 . This implies that the charge is not dominated by a finite set of multipoles, but rather appears to be consistent with a self-similar charge distribution. Moreover, although the charge saturates after about 25 rubbings, the distribution remains self-similar during the charging phase as well as after saturation.

Second, we visualize the charge distributions by exposing charged balloons to a cloud of charged toner, which adheres to oppositely charged regions on the balloons. To achieve this, in Fig. 2(a), we have cleaned two balloons with alcohol and, after drying we have rubbed the balloons together in a symmetric circular motion through 30 revolutions. Thereafter, we have exposed the balloons to a cloud of magenta toner (details in Materials and Methods) that is generated by shaking a small quantity of toner in a metal can beneath each balloon; shaking causes the toner to rub against the can, thus charging the toner. Measurements using the voltage probe indicate that the toner becomes positively charged, so the regions of the balloon to which toner adheres are negative. Two distinct patterns are boxed in Fig. 2(a): elliptical spots on the balloon on the left, and striations on the balloon on the right. These patterns appear reproducibly, with the spots tending to form on balloons that are net positively charged, and striations on balloons that are negatively charged.

Finer details of these patterns can be revealed by exposing the balloons to "bipolar toner" consisting of a blend of two different types of toner (see Materials and Methods): when these toners rub against one another, they can charge oppositely and so can be used to identify both positive and negative charges on a surface. Characteristic results are shown for two balloons in Fig's 2(b) (for a positive balloon) and 2(c) (for a negative balloon). We find that spotted and striated patterns are often intermixed, with a single balloon exhibiting both types of patterns (cf. Fig. 2(a)). While we cannot discount the possibility that these patterns - and the charging phenomenon in general - are affected by manufacturing variations between the balloons, typically one pattern or the other will dominate on a single balloon, and the choice of pattern on the same balloon can change seemingly arbitrarily following cleaning. This leads us to believe that an intrinsic charging instability may be at work, with the spot pattern being characteristic of net positive charge, and the branched striations of net negative charge. Moreover, since both spots and 
branches can be associated in other contexts with self-similar, recursive processes ${ }^{9}$, we seek to determine whether the process underlying these unanticipated patterns may likewise be recursive.
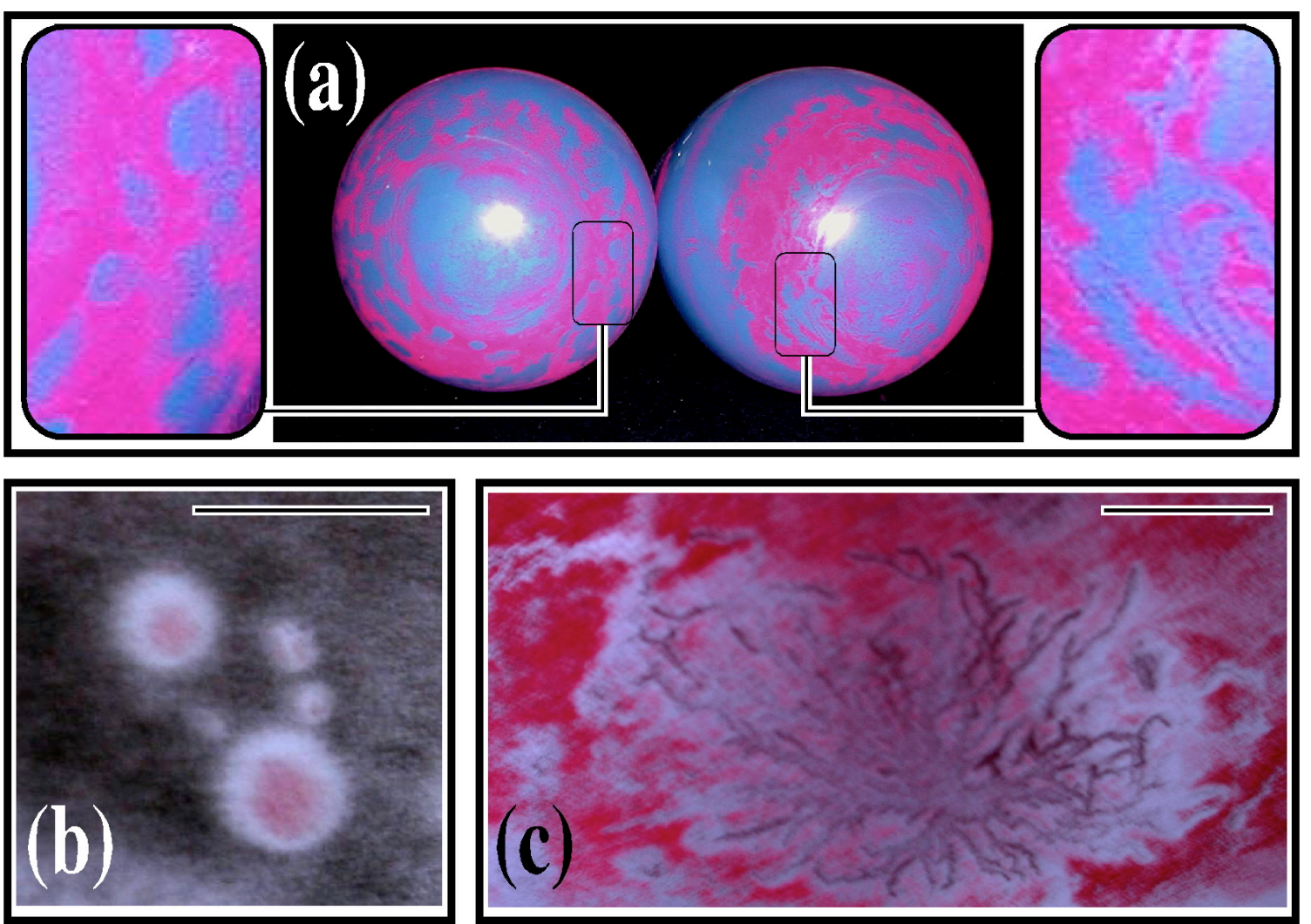

Figure 2 - (a) Two latex balloons rubbed against one another, in this case in a symmetrical circular motion, and exposed to charged magenta toner. Toner is positively charged, so locations where it sticks are negatively charged. Notice that the left balloon's charge patterns are dominated by elliptical spots (leftmost enlargement), while the right balloon is dominated by streaks (rightmost enlargement). (b) Enlarged view of characteristic spot pattern on positively charged balloon. (c) Enlarged view of branched pattern on negatively charged balloon. Balloons in (b) and (c) are from a fresh pair that are cleaned, then rubbed against one another, and finally exposed to 'bipolar' toner. Length bars are approximately $1 \mathrm{~cm}$.

To achieve this in a more controlled setting than manual rubbing of common balloons, we turn to simulations in which all details can be prescribed. To construct this simulation, we note from Fig. 2(b) that the centers of the charged spots are not symmetric, but are eccentrically placed toward about 5 o'clock, while at 10 and 12 o'clock, smaller spots seem to radiate away from the lower spot. This suggests that the toner-identified charges may have been dragged by rubbing past fixed spots from 5:00 toward 11:00. We hypothesize that rubbing may by some mechanism cause smaller like-charged spots to be shed downstream: such a mechanism applied to ever-smaller spots is recursive and could be a candidate mechanism to generate a self-similar charge distribution.

We test whether such a mechanism may occur by particle-dynamics simulation applied to the problem of a fixed spot of charged particles. The simulation is performed in $2 \mathrm{D}$, meant to mimic the 
surface of a balloon, and charged particles, representing free surface ions, are accelerated with a uniform force per unit mass, representing a steady force due to rubbing, past the fixed spot. Details of particledynamics methods appear elsewhere ${ }^{10}$ : in synopsis this model uses identically sized, elasto-plastic particles with kinetic sliding friction, and permits particles to interact electrostatically with inverse $r^{2}$ force as if their entire assigned charge were localized at the particles' centers. Particles are randomly placed upstream of the spot with charges chosen to be either positive or negative with equal probability. We reason that if simple balloons exhibit charge amplification, then the effect may be robust, and so the charges used in the simulation are somewhat arbitrary (see Fig. 3(a) legend): we have varied both spot and particle charges by a factor of 2 and obtain similar results.

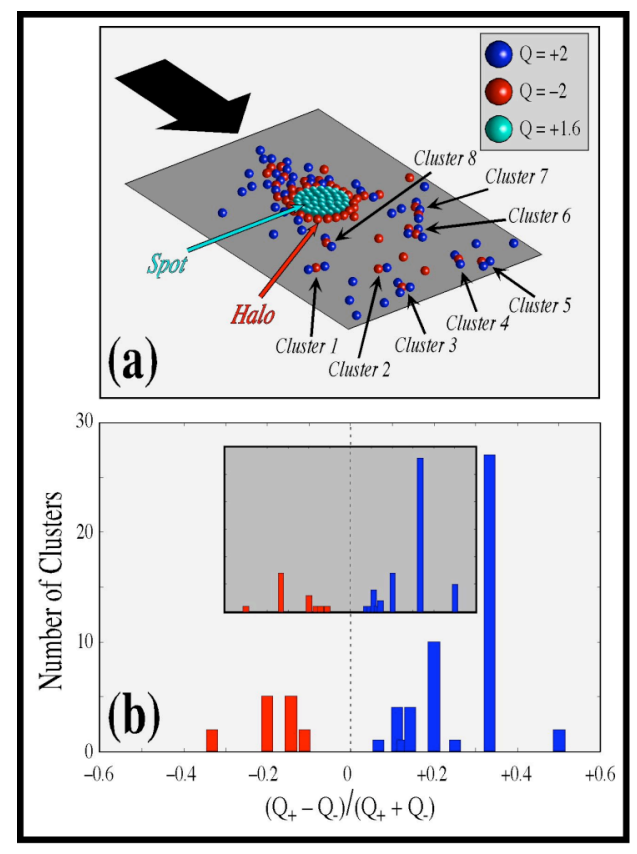

Figure 3 - Simulations of the formation of charged clusters downstream of a charged spot. (a) Positive (blue) and negative (red) charged particles advected past a fixed charged spot (green) in the direction of the black arrow. The particle advection is meant to mimic rubbing by another surface in that direction. Charge magnitudes are shown in the legend in computational units. Clusters identified are all positively charged except for cluster 2, which is neutral. (b) Distributions of downstream charged clusters in simulations of 250,000 timesteps; blue bars are positively charged, red bars are negative. Main plot: distribution seen using fixed timestep, $\Delta t$, where cluster charges are determined when clusters reach 20 particle diameters downstream of fixed spot. Inset plot: distribution using a timestep of $2 \Delta t$ and where cluster charges are evaluated every 5000 timesteps.

The configuration of charges is shown in Fig. 3(a) after 60,000 timesteps, where one new particle is generated upstream every 500 timesteps. The direction of constant acceleration of the particles is indicated by the large arrow, and the fixed charged spot is shown in green. A red halo of opposite charges indicated forms spontaneously and remains intact throughout the simulations, although individual particles in the halo become washed downstream by the constant imposed acceleration. We propose that the halo 
may paradoxically attract positive particles (blue), causing these particles to be shed downstream as shown in the figure, while negative particles (red) are repelled and travel as lone charges.

We record the positions of all particles every 5000 timesteps for later analysis. From this data record, we evaluate how many clusters of 2 or more particles are positively and negatively charged using a positively charged fixed spot. We are interested in net charging, and so neutral clusters (which are plentiful) are ignored. Fig. 3(a) shows 8 clusters, of which all but cluster 2 are positively charged. This example is fortuitous: at most times, somewhat fewer clusters are positively charged. The numbers and charges of clusters are evaluated in Fig. 3(b), which shows the total charges on clusters downstream of the fixed spot from two simulations of 250,000 timesteps. The clusters evolve over time, and so we evaluate cluster charges in two ways. In the main plot we show cluster charges evaluated when a cluster first reaches a 'finish line' 20 particle diameters downstream of the spot center, while in the inset (using the same axes), we evaluate the charge on every cluster every 5000 timesteps. Thus in the second case, we count clusters more than once as they travel downstream; furthermore to confirm that these results are not mere computational artifacts, we double the timestep in the simulation shown in the inset (and we therefore spawn new upstream particles twice as often: every 250 timesteps). In both cases we obtain similar results: in the main plot we find that 50/64 clusters are positively charged and in the inset we find 49/63 clusters are positive.

These simulations suggest that charged spots shed smaller charged clusters that are predominantly (about 75\%) of the same sign. Since charge is conserved, it follows that oppositely charged particles must be unattached. It is observed in experiments that balloons emit a crackling sound during rubbing ${ }^{11}$, and it seems likely that this may be caused by the discharge of isolated charges to the opposing balloon, which leave less mobile clusters of charges attached to the originating balloon. This conjecture remains for future investigation, as does the natural speculation that the production of smaller spots from larger ones - as well as discharge patterns formed following discharges - may both produce the measured self-similar charge distributions. 


\section{Conclusion:}

In conclusion, we have seen that very common materials - latex balloons - can tribocharge when symmetrically rubbed against one another, despite lacking either an obvious source for charge carriers or an apparent triboelectric gradient to drive the charge transfer. We have found both by quantitative measurement of voltage as a function of distance and by qualitative examination of the patterns on charged balloons that the charges that accumulate appear to be complex and may be consistent with a recursive, self-similar, model for charge formation. We have proposed and confirmed by direct simulation that once a fixed spot of charge forms on an insulating surface, an oppositely charged halo appears to catalyze the formation of downstream smaller clusters of similar charge to the original spot. We have conjectured that this may be the source of the self-similar charge distributions seen.

These results are easily reproduced, but leave much to be understood. Foremost among the open questions is how insulators recruit the free charges that manifestly must be present during tribocharging. The answer to this, very basic, question remains elusive, however to inform future investigation, we have performed a final set of experiments to establish the extent to which the charges may be recruited from free ions in the air. The species of airborne ions have been identified elsewhere ${ }^{12}$, and are found to include higher mobility anions, such as $\mathrm{O}^{-}$and $\mathrm{O}_{3}^{-}$, alongside lower mobility cations such as $\left(\mathrm{NO}_{\mathrm{x}}^{+}\right)\left(\mathrm{H}_{2} 0\right)_{\mathrm{n}}$. It seems plausible that the lower mobility cations may contribute to predominantly positive spots (Fig. 2(b)) through a mechanism such as that described in Fig. 3, while predominantly negatively charged striations (Fig. 2(c)) may be produced by discharges of higher mobility anions ${ }^{12}$.

To assess this possibility, we inflated identical balloons using helium, which is inert and lacks these identified species, and we rubbed these balloons together in air and in an atmosphere of the inert gas. We found in multiple separate trials that balloons readily charge if filled with inert gas and rubbed in the presence of air, however the same balloons filled either with air or with inert gas do not tribocharge when rubbed against one another in an inert atmosphere. Triboelectrification of dissimilar materials is not strongly affected by the surrounding gas, thus we have confirmed that the balloons continue to be readily charged by rubbing with paper or cotton. The presence or absence of triboelectrification was established by determining whether balloons attract one another and was confirmed by direct measurements using the voltmeter probe. These experiments were performed in an inverted 15 gallon tank that traps the helium. The lack of tribocharging between identical materials in an inert atmosphere suggests that the recruitment of free charges by insulators occurs either as a result of the air itself (e.g. following dissociation of air ions during discharges) or by alteration of polymeric surface states by air molecules. In either case, it appears that the interaction between the surface and air plays a significant, but yet to be clarified, role in the tribocharging instabilities that we have studied. 


\section{Materials and methods:}

Prior to experimentation, we prepare the balloons by cleaning them with ethanol and paper towels; we suspend the balloons by the nipple away from contact with any other surface during drying. Once the balloons have air dried, we hold the nipple-ends of each balloon, point the opposite ends of the balloons toward one another, and rub them together in a symmetric, circular motion. The balloons used are "helium quality" natural latex rubber (Unique Industries, Philadelphia, PA); for voltage and bipolar toner data we use white iridescent balloons; for Fig's 1(a) and 2(a), we use colored balloons for visual contrast. We measure the charges on the balloons using a null voltmeter probe (Trek Inc., Model 344 with probe 6000B7C, Medina, NY) attached to a fixed stand distant from other charges and conductors, and we record both the voltage and the distance using a video camera that is placed so as to record both the balloon's position against a backdrop containing a ruled measure and the voltmeter output display. In this way, we can simultaneously reconstruct the balloon position and the voltmeter output from the video recording. The magenta toner used is commercially available (Samsung model CLP-M300A); the bipolar toner is this toner mixed in equal parts with black toner (Xerox model 6R881).

\section{Acknowledgments:}

This work was supported in part by the NSF ERC on Structured Organic Particulate Systems (CSOPS), grant \# EEC-0540855. Additional support was provided by Trek Inc. We thank David Maiullo and Maciej Noras for help and advice. 


\section{References}

$1 \quad$ W. Gilbert, De Magnete (Dover, NY 1991) 89 -97 Translated by P. Fleury Mottelay

2 JT Desaguliers, “Some further observations concerning electricity,” Philo. Trans. 42 (1742) 14-8

3 W. Thomson, "On a self-acting apparatus for multiplying and maintaining electric charges, with applications to illustrate the Voltaic theory," Proc. R. Soc. Lond. 16 (1867) 67-72

$4 \quad$ WR Harper, Contact and Frictional Electrification (Clarendon, Oxford, 1967)

5 J. Lowell \& WS Truscott, “Triboelectrification of identical insulators. I. An experimental investigation” J. Phys. D 19 (1986) 1273-80

$6 \quad$ DS Schmidt, RA Schmidt \& JD Dent, "Electrostatic force on saltating sand” J. Geophys. Res. 103 (1998) 8897 9001

$7 \quad$ P. Lorrain \& DR Corson, Electromagnetic fields and waves, (WH Freeman \& Co, San Francisco, 1970) 65-6

$8 \quad$ PSH Henry, "The role of asymmetric rubbing in the generation of static electricity" J. Appl. Phys, 4 (1953) S31-36

$9 \quad$ P. Ball The self-made tapestry: pattern formation in nature, (Oxford U. Press, Oxford, 1999)

10 HJ Herrmann, “Simulation of granular media,” Physica A 191 (1992) 263-76.

11 T. Shinbrot, "Triboelectrification and Razorbacks: geophysical patterns produced in dry grains," Phys. Rev. Lett. 96 (2006) 178002 1-4

12 MM Shahin, "Nature of charge carriers in positive and negative corona discharges," Appl Optics Suppl. Electrophotography, 3 (1969) 106-10. 